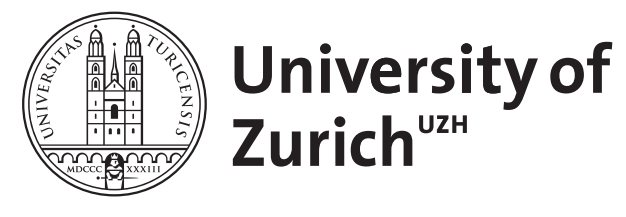

\title{
Energy extraction from black holes
}

Straumann, N

\begin{abstract}
In this lecture I give an introduction to the rotational energy extraction of black holes by the electromagnetic Blandford-Znajek process and the generation of relativistic jets. After some basic material on the electrodynamics of black hole magnetospheres, we derive the most important results of Blandford and Znajek by making use of Kerr-Schild coordinates, which are regular on the horizon. In a final part we briefly describe results of recent numerical simulations of accretion flows on rotating black holes, the resulting large-scale outflows, and the formation of collimated relativistic jets with high Lorentz factors
\end{abstract}

DOI: https://doi.org/10.1063/1.2902800

Posted at the Zurich Open Repository and Archive, University of Zurich ZORA URL: https://doi.org/10.5167/uzh-16770

Book Section

Accepted Version

Originally published at:

Straumann, N (2008). Energy extraction from black holes. In: Macias, A; Lämmerzahl, C; Camacho, A. Recent developments in gravitation and cosmology. 3rd Mexican Meeting on Mathematical and Experimental Physics. Melville NY: American Institut of Physics, 75-93.

DOI: https://doi.org/10.1063/1.2902800 


\title{
ENERGY EXTRACTION FROM BLACK HOLES
}

\author{
Norbert Straumann \\ Institute for Theoretical Physics, University of Zurich, \\ Winterthurerstrasse 190, CH-8057 Zurich, Switzerland
}

\begin{abstract}
In this lecture I give an introduction to the rotational energy extraction of black holes by the electromagnetic Blandford-Znajek process and the generation of relativistic jets. After some basic material on the electrodynamics of black hole magnetospheres, we derive the most important results of Blandford and Znajek by making use of Kerr-Schild coordinates, which are regular on the horizon. In a final part we briefly describe results of recent numerical simulations of accretion flows on rotating black holes, the resulting large-scale outflows, and the formation of collimated relativistic jets with high Lorentz factors.
\end{abstract}

Keywords: black hole physics - MHD - galaxies:jets - relativity

PACS: 04.70.-s, 52.30.Cv, 97.60.Lf

\section{INTRODUCTION}

For good reasons it is by now generally believed that active galactic nuclei (AGNs) are powered by supermassive black holes (BHs), with typical masses of about $10^{9} M_{\odot}$. Indeed, this seems to be the only way to generate the enormous energy of order $10^{46} \mathrm{erg} / \mathrm{s}$ in regions not much larger than the solar system. Accretion of matter by black holes, offers the most efficient power supply. A few solar masses of gas per year suffice to power the most energetic quasars.

It is known for long that AGNs often expel enormous energies in two oppositely directed relativistic jets with Lorentz factors $\gamma \sim 10$. More recently, moderately large Lorentz factors ( $\gamma$ a few) have been seen in BH x-ray binaries (XRBs), called microquasars. Relativistic jets have also been observed in gamma-ray bursts (GRBs). How are these remarkable jets formed? Which mechanisms are responsible for the concentrated energy input at their origin? Most probably processes connected to BHs are involved. Among these, perhaps the most promising possibility is the energy extraction from a black hole via magnetic fields, since fairly strong magnetic fields are likely to be present in accretion flows on the central BH. This scenario, which gives rise to a long-range coherence, is the main subject of this talk. Basic questions to be answered are: Which forces drive the jets? What are the mechanisms that regulate their content, and how are they collimated?

Penrose first discovered that a substantial fraction of a Kerr black hole mass can be converted, at least in principle, into the energy of surrounding matter or radiation. However, the Penrose process is inefficient under typical astrophysical conditions. Later it was found that external electromagnetic fields can be used to extract rotational energy of black holes. Of great influence was a pioneering study by Blandford \& Znajek [8] 
which demonstrated the possibility of an electromagnetically driven wind from a rotating black hole, provided the space around the black hole is filled with plasma. In their paper they developed a general theory of force-free steady-state axisymmetric magnetospheres of black holes, and estimated that the power of the wind could be high enough to explain the energetics of radio galaxies and quasars.

This work triggered the so called membrane model by Thorne and collaborators [1] (see also [7]). In this model one first splits the elegant 4-dimensional physical laws of general relativity (GR) into space and time $(3+1$ splitting). For a general situation this can be done in many ways (reflecting the gauge freedom in GR) since there is no canonical fibration of spacetime by level surfaces of constant time. (For a stationary BH we shall choose foliations which are adapted to the corresponding Killing field.) Relative to these the dynamical variables (electromagnetic fields, etc) become quantities on an absolute space which evolve as functions of an absolute time, as we are accustomed to from non-relativistic physics. We shall see, for example, that the $3+1$ splitting brings Maxwell's equations into a form which resembles the familiar form of Maxwell's equations for moving conductors. We can then use the pictures and our experience from ordinary electrodynamics.

In a second more specific step one replaces the boundary conditions at the horizon by physical properties (electric conductivity, etc) of a fictitious membrane. This procedure is completely adequate as long as one is not interested in fine details very close to the horizon. The details of this boundary layer are, however, completely irrelevant for astrophysical applications. (The situation is similar to many problems in electrodynamics, where one replaces the real surface properties of a conductor and other media by idealized boundary conditions.)

This approach turns the drawback of the Boyer-Lindquist coordinates, which become singular at the horizon, into an advantage. The membrane model has the merit that it allows us to understand astrophysical processes near black holes more easily, because things become then closer to the intuition we have gained from other fields of physics, for instance from the electrodynamics of moving bodies. However, this membrane reformulation of regularity requirements at the horizon - in terms of the singular BL coordinates - is clearly artificial. More importantly, it hides the fact that the key role in the electrodynamic Blandford-Znajek mechanism is played not by the black hole event horizon, but by its ergo-sphere (as in the Penrose process). Later I shall present an alternative derivation of the most important results of Blandford and Znajek, in making use of Kerr-Schild coordinates. This foliation is not singular at the horizon, whence no boundary problems arise.

Kerr-Schild coordinates not only simplify theoretical analysis, but have also successfully been used in some numerical simulations. In recent years several codes have been developed to study the magnetic energy extraction from BHs. In particular, various groups have addressed the question how relativistic jets may have formed. In the last part of the lecture I will briefly report on what has been done and achieved so far in numerical studies of what is now often dubbed the (generic) "Blandford-Znajek mechanism", although only a specific version was originally proposed.

We begin with some basic material on black hole electrodynamics. 


\section{SPACE-TIME SPLITTING OF ELECTRODYNAMICS}

Let us perform the $3+1$ splitting of the general relativistic Maxwell equations on a stationary spacetime $\left(M,{ }^{(4)} \mathbf{g}\right)$. Most of what follows could easily be generalized to spacetimes which admit a foliation by spacelike hypersurfaces (see, e.g., Ref. [2]), but this is not needed in what follows. Similar $3+1$ decompositions can be carried out for the other equations of general relativistic magnetohydrodynamics (GRMHD) .

Slightly more specifically, we shall assume that globally $M$ is a product $\mathbf{R} \times \Sigma$, such that the natural coordinate $t$ of $\mathbf{R}$ is adapted to the Killing field $k$, i.e., $k=\partial_{t}$. We decompose the Killing field into normal and parallel components relative to the "absolute space" $(\Sigma, \mathbf{g})$, $\mathbf{g}$ being the induced metric on $\Sigma$,

$$
\partial_{t}=\alpha u+\beta .
$$

Here $u$ is the unit normal field and $\beta$ is tangent to $\Sigma$. This is what one calls the decomposition into lapse and shift; $\alpha$ is the lapse function and $\beta$ the shift vector field. We shall usually work with adapted coordinates $\left(x^{\mu}\right)=\left(t, x^{i}\right)$, where $\left\{x^{i}\right\}$ is a coordinate system on $\Sigma$. Let $\beta=\beta^{i} \partial_{i} \quad\left(\partial_{i}=\partial / \partial x^{i}\right)$, and consider the basis of 1 -forms

$$
\alpha d t, \quad d x^{i}+\beta^{i} d t .
$$

One verifies immediately, that this is dual to the basis $\left\{u, \partial_{i}\right\}$ of vector fields. Since $u$ is perpendicular to the tangent vectors $\partial_{i}$ of $\Sigma$, the 4-metric has the form

$$
{ }^{(4)} \mathbf{g}=-\alpha^{2} d t^{2}+g_{i j}\left(d x^{i}+\beta^{i} d t\right)\left(d x^{j}+\beta^{j} d t\right),
$$

where $g_{i j} d x^{i} d x^{j}$ is the induced metric $\mathbf{g}$ on $\Sigma$. Clearly, $\alpha, \beta$, and $\mathbf{g}$ are all timeindependent quantities on $\Sigma$.

We introduce several kinds of electric and magnetic fields. Obviously, the spatial 1forms

$$
\check{\mathscr{E}}:=-i_{k} F, \quad \check{\mathscr{H}}:=i_{k} * F,
$$

have an intrinsic meaning. If we set

$$
F=\mathscr{B}+\check{\mathscr{E}} \wedge d t, \quad * F=\mathscr{D}-\check{\mathscr{H}} \wedge d t,
$$

the electric and magnetic 2 -forms $\mathscr{D}, \mathscr{B}$ are spatial. Two further electric and magnetic 1-forms are defined by

$$
\mathscr{E}=\star \mathscr{D}, \quad \mathscr{H}=\star \mathscr{B},
$$

where $\star$ denotes the spatial Hodge dual on $(\Sigma, \mathbf{g})$. The corresponding vector fields are denoted by $\vec{E}$ and $\vec{B}$. These are measured by observers moving with 4-velocity $u$, socalled FIDOs, for fiducial observers. One easily finds the following algebraic relations:

$$
\check{\mathscr{E}}=\alpha \mathscr{E}-i_{\beta} \mathscr{B}, \quad \check{\mathscr{H}}=\alpha \mathscr{H}+i_{\beta} \mathscr{D} .
$$

We give also the coordinate components of the various fields:

$$
\check{\mathscr{E}}_{i}=-F_{t i}, \quad \check{\mathscr{H}}_{i}=* F_{t i}=\frac{\alpha}{2} \eta_{i j k} F^{j k}, \quad \mathscr{B}_{i j}=F_{i j}, \quad B^{i}=\frac{1}{2} \eta^{i j k} F_{j k}, \quad \mathscr{D}_{i j}=* F_{i j}, \quad E^{i}=\alpha F^{t i}
$$


where $\eta_{i j k}$ denotes the Levi-Civita tensor on $(\Sigma, \mathbf{g})$.

In terms of the current 4-vector $J^{\mu}$ the electric charge density is $\rho_{e l}=\alpha J^{t}$. Beside the spatial current 1 -form $j=J_{k} d x^{k}$ we use also its spatial Hodge dual $\mathscr{J}=\star j$. Using also $\hat{\rho}=\star \rho_{e l}$, the Maxwell equations can be written in $3+1$ form as (d denotes the exterior differential on $\Sigma$ ):

$$
\begin{aligned}
\mathbf{d} \mathscr{B}=0, & \partial_{t} \mathscr{B}+\mathbf{d} \check{\mathscr{E}}=0, \\
\mathbf{d} \mathscr{D}=4 \pi \hat{\rho}, & -\partial_{t} \mathscr{D}+\mathbf{d} \check{\mathscr{H}}=4 \pi \check{\mathscr{J}}, \quad \check{\mathscr{J}}:=\alpha \mathscr{J}-i_{\beta} \hat{\rho} .
\end{aligned}
$$

These can be translate into a vector analytic form. (For this as well as detailed derivations, see [7].) All this looks like Maxwell's equations for moving conductors.

\section{Integral Formulas}

As is well-known from ordinary electrodynamics, it is often useful to write the basic laws in integral forms. Consider, for instance, the induction law in (9). If we integrate this over a surface area $\mathscr{A}$, which is at rest relative to the absolute space, we obtain with Stokes' theorem $(\mathscr{C}:=\partial \mathscr{A})$, using also (7),

$$
\oint_{\mathscr{C}} \alpha \mathscr{E}=-\frac{d}{d t} \int_{\mathscr{A}} \mathscr{B}+\oint_{\mathscr{C}} i_{\beta} \mathscr{B}
$$

The left hand side is the electromotive force $(\mathrm{EMF})$ along $\mathscr{C}$. The last term is similar to the additional term one encounters in Faraday's induction law for moving conductors. It is an expression of the coupling of $\mathscr{B}$ to the gravitomagnetic field and plays a crucial role in much that follows. This term contributes also for a stationary situation, for which (10) reduces to

$$
\operatorname{EMF}(\mathscr{C})=\oint_{\mathscr{C}} \alpha \mathscr{E}=\oint_{\mathscr{C}} i_{\beta} \mathscr{B}
$$

\section{BLACK HOLE IN A HOMOGENEOUS MAGNETIC FIELD}

As an instructive example and a useful tool we discuss now an exact solution of Maxwell's equations in the Kerr metric, which becomes asymptotically a homogeneous magnetic field. This solution can be found in a strikingly simple manner [3].

For any Killing field $K$ and its 1 -form $K^{b}$ one has the following identity $\delta d K^{b}=$ $2 R(K)$, where $\delta$ denotes the co-differential and $R(K)$ is the 1-form with components $R_{\mu \nu} K^{v}$. In components this is equivalent to

$$
K_{\mu ; \alpha}^{; \alpha}=-R_{\mu \alpha} K^{\alpha}
$$

This form can be obtained by contracting the indices $\sigma$ and $\rho$ in the following general equation for a vector field $\xi_{\sigma ; \rho \mu}-\xi_{\sigma ; \mu \rho}=\xi_{\lambda} R_{\sigma \rho \mu}^{\lambda}$ and by using the consequence $K_{; \sigma}^{\sigma}=0$ of the Killing equation $K_{\sigma ; \rho}+K_{\rho ; \sigma}=0$. For a vacuum spacetime we thus have $\delta d K^{b}=0$ for any Killing field. Hence, the vacuum Maxwell equations are satisfied if $F$ is a constant linear combination of the differential of Killing fields (their duals, to be precise). For the Kerr metric, as for any axially symmetric stationary spacetime, 
we have two Killing fields $k$ and $m$. Both in Boyer-Lindquist (BL) and in Kerr-Schild (KS) foliations the Killing fields are coordinate derivatives: $k=\partial_{t}$ and $m=\partial_{\varphi}\left(k^{b}\right.$ and $m^{b}$ denote the corresponding 1-forms). The Komar formulae provide convenient expressions for the total mass $M$ and the total angular momentum $J$ of the Kerr $\mathrm{BH}$ :

$$
M=-\frac{1}{8 \pi} \int_{\infty} * d k^{b}, \quad J=\frac{1}{16 \pi} \int_{\infty} * d m^{b}
$$

(for $G=1$ ).

We try the ansatz

$$
F=\frac{1}{2} B_{0}\left(d m^{b}+2 a d k^{b}\right) \quad\left(B_{0}=\text { const }\right),
$$

and choose $a$ such that the total electric charge

$$
Q=-\frac{1}{4 \pi} \int_{\infty} * F
$$

vanishes. The Komar formulae (13) tell us that

$$
Q=-\frac{1}{8 \pi} B_{0}(16 \pi J-2 a \cdot 8 \pi M)
$$

and this vanishes if $a=J / M$ (which is the standard meaning of the symbol $a$ in the Kerr solution).

Clearly, $F$ is stationary and axisymmetric:

$$
L_{k} F=L_{m} F=0,
$$

because (dropping $b$ from now on) $L_{k} d k=d L_{k} k=0,\left(L_{k} k=[k, k]=0\right)$, etc.

The solution (14) can be expressed in terms of a potential: $F=d A$, with

$$
A=\frac{1}{2} B_{0}(m+2 a k)=\frac{1}{2} B_{0}\left(g_{\mu \varphi}+2 a g_{\mu t}\right) d x^{\mu},
$$

where the last expression holds in Boyer-Lindquist (BL) as well as in Kerr-Schild (KS) coordinates. Asymptotically, this describes a magnetic field in the z-direction whose magnitude is $B_{0}$.

It is straightforward to work out explicit expressions for $\vec{E}$ and $\vec{B}$. The electric field has a quadrupole-like structure and is poloidal. It is proportional to $a$, and thus due to the gravitomagnetic component of the Kerr solution. Its emergence is of great astrophysical significance.

It is of interest to work out the magnetic flux through the equator of the $\mathrm{BH}$, i.e.,

$$
\Phi=\int_{\text {upper h. }} \mathscr{B}=\int_{\text {equator }} \mathscr{A}=2 \pi \mathscr{A} \varphi \mid \text { equator }
$$

One finds

$$
\Phi=4 \pi B_{0} M\left(r_{H}-M\right)=4 \pi B_{0} M \sqrt{M^{2}-a^{2}} .
$$


Generically one has, as expected, $\Phi \approx \pi r_{H}^{2} B_{0}$. Note, however, that (20) vanishes for an extremal $\mathrm{BH}(a=M)$. In other words, the flux is completely expelled from the black hole, like in the Meissner-Ochsenfeld effect in superconductivity. In order to study the structure the Wald solution close to the black hole one has to express it in terms of KerrSchild coordinates (see Sect. 6). The magnetic field lines in these coordinates are shown for $a=M$ in Fig. 1, taken from [4].

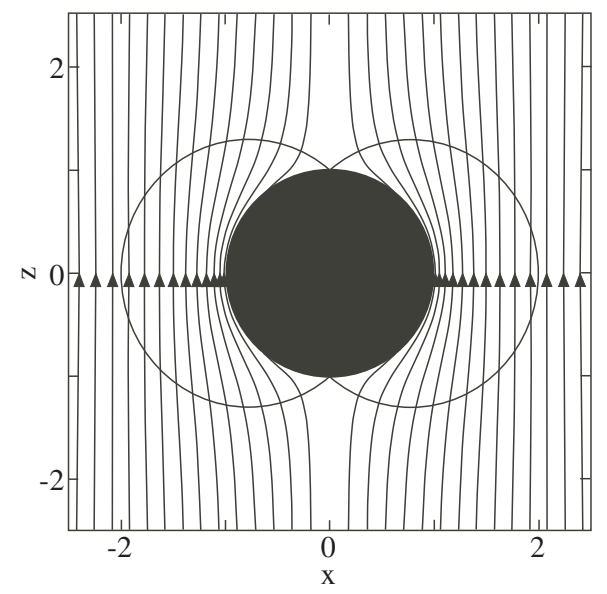

FIGURE 1. Black hole "Meissner effect" (from [4]). The magnetic field lines of Wald's vacuum solution in KS coordinates for $a=1$ are shown in the $x=r \sin \vartheta, z=r \cos \vartheta$ plane. The partial circles (thick lines) bound the ergosphere.

\section{AXISYMMETRIC STATIONARY FIELDS}

In this section we discuss some consequences of Maxwell's equations for a stationary axisymmetric magnetoshere outside a black hole.

\subsection{Potential Representation}

For an axisymmetric field we have $L_{\partial_{\varphi}} \mathscr{B}=0 \leftrightarrow \mathbf{d} i_{\partial_{\varphi}} \mathscr{B}=0$, whence $i_{\partial_{\varphi}} \mathscr{B}=$ $-\mathbf{d} \Psi / 2 \pi$. From this we conclude that for the BL foliation $\mathscr{B}$ can be expressed in terms of two potentials $\Psi$ and $I$,

$$
\mathscr{B}=\underbrace{\frac{1}{2 \pi} \mathbf{d} \Psi \wedge \mathbf{d} \varphi}_{\text {poloidal part }}+\underbrace{\frac{2 I}{\alpha} * \mathbf{d} \varphi}_{\text {toroidal part }},
$$

both of which can be taken to be independent of $\varphi$. (The physical meaning if $I$ will be discussed further below.) $\Psi$ is the magnetic flux function (see Fig. 2), because the poloidal flux inside a tube $\{\Psi=$ const $\}$ is

$$
\int \mathscr{B}=\frac{1}{2 \pi} \int \mathbf{d}(\Psi \mathbf{d} \varphi)=\frac{1}{2 \pi} \oint \Psi \mathbf{d} \varphi=\Psi, \quad \Psi(0)=0 .
$$


$\Psi$ is constant along magnetic field lines, as should be clear from Fig. 2. It is easy to

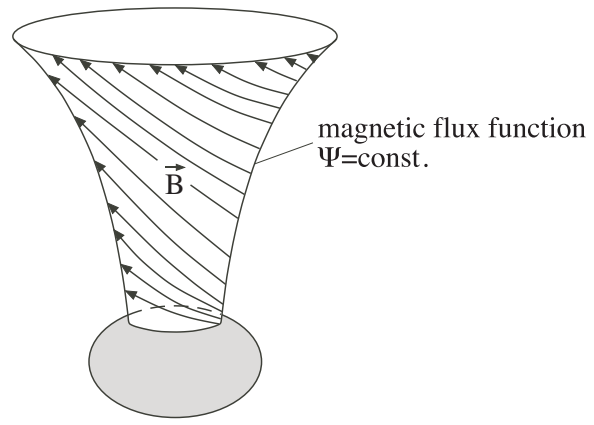

FIGURE 2. Axisymmetric magnetic field. The total flux inside the magnetic surface defines the flux function $\Psi$.

show this also formally.

The electric field $\vec{E}$ has no toroidal component for an axisymmetric stationary situation. This is an immediate consequence of the induction law: Applying (10) for a stationary and axisymmetric configuration to the closed integral curve $\mathscr{C}$ of the Killing field $\partial_{\varphi}$, we obtain

$$
\oint_{\mathscr{C}} \alpha \mathscr{E}=\oint_{\mathscr{C}} i_{\beta} \mathscr{B}=\frac{\omega}{2 \pi} \oint_{\mathscr{C}} \mathbf{d} \Psi=0 \Longrightarrow \vec{E}^{\text {tor }}=0
$$

A similar application of Ampère's law in integral form gives

$$
\oint_{\mathscr{C}} \alpha \mathscr{H}=4 \pi \int_{\mathscr{A}} \alpha \mathscr{J}=4 \pi I
$$

where $I$ is the total upward current through a surface $\mathscr{A}$ bounded by $\mathscr{C}$. This shows that the potential $I$ in (21) is the upward current.

So far we did not make any model assumptions about the physics of the magnetosphere. But now we assume that the electromagnetic field is degenerate, i.e., that $\vec{E} \cdot \vec{B}=0$, which is equivalent to the invariant statement $F \wedge F=0$. (This is satisfied for ideal GRMHD and for force-free fields; see Sects. 5 and 6.) Because $\mathscr{E}$ is poloidal, we can then represent the electric field as follows

$$
\mathscr{E}=i_{\vec{v}_{F}} \mathscr{B} \quad\left(\vec{E}=-\vec{v}_{F} \times \vec{B}\right),
$$

where $\vec{v}_{F}$ is toroidal. Let us set

$$
\vec{v}_{F}=: \frac{1}{\alpha}\left(\Omega_{F}-\omega\right) \tilde{\omega} \vec{e}_{\varphi}
$$

For the interpretation of $\Omega_{F}$ note the following: For an observer, rotating with angular velocity $\Omega$, the 4-velocity is $u=u^{t}\left(\partial_{t}+\Omega \partial_{\varphi}\right)$. On the other hand, $u=\gamma\left(e_{0}+\vec{v}\right)$, where $\vec{v}$ is the 3-velocity relative to a FIDO. Using also $\partial_{t}=\alpha e_{0}+\vec{\beta}$ we get $\Omega \vec{\partial}_{\varphi}=\alpha \vec{v}-\vec{\beta}$ or

$$
\vec{v}=\frac{1}{\alpha}(\Omega-\omega) \vec{\partial}_{\varphi}=\frac{1}{\alpha}(\Omega-\omega) \tilde{\omega} \vec{e}_{\varphi}
$$


This has the same form as (26). Since the transformed electric field $\vec{E}^{\prime}=\gamma_{F}\left(\vec{E}+\vec{v}_{F} \times \vec{B}\right)$ vanishes, we may regard $\Omega_{F}$ as the angular velocity of the magnetic field lines. (The transformed frame can be defined as the "local rest frame" of the magnetic field lines.) Eqs. (25) and (26) imply

$$
\alpha \mathscr{E}=-\frac{\Omega_{F}-\omega}{2 \pi} \mathbf{d} \Psi, \quad \check{\mathscr{E}}=-\mathbf{d} \Psi
$$

thus $\vec{E}$ is perpendicular to the surfaces $\{\Psi=$ const $\}$. Taking the exterior derivative, and using induction law, we get $\mathbf{d} \Omega_{F} \wedge \mathbf{d} \Psi=0 \Longrightarrow \Omega_{F}=\Omega_{F}(\Psi)$. So the electromagnetic field is determined in terms of $\Psi, I$ and the "flow (field line) constant" $\Omega_{F}(\Psi)$.

\subsection{EMF outside a rotating Black Hole}

In Fig. 3 we consider a stationary rotating $\mathrm{BH}$ in an external magnetic field (like in $\S 3)$. The integral in (11) along the field lines gives no contribution and far away $\beta$ drops rapidly $\left(\sim r^{-2}\right)$. Thus, there remains only the contribution from the horizon $\left(\mathscr{C}_{H}\right)$ of the path $\mathscr{C}$ in Fig. 3:

$$
\mathrm{EMF}=\int_{\mathscr{C}_{H}} i_{\beta_{H}} \mathscr{B}, \quad \beta_{H}=-\Omega_{H} \partial_{\varphi}
$$

where $\Omega_{H}$ is the angular velocity of the horizon (only the normal component $\vec{B}_{\perp}$ contributes). I recall that $\Omega_{H}=a\left(2 M r_{H}\right)^{-1}, r_{H}=M+\sqrt{M^{2}-a^{2}}$.

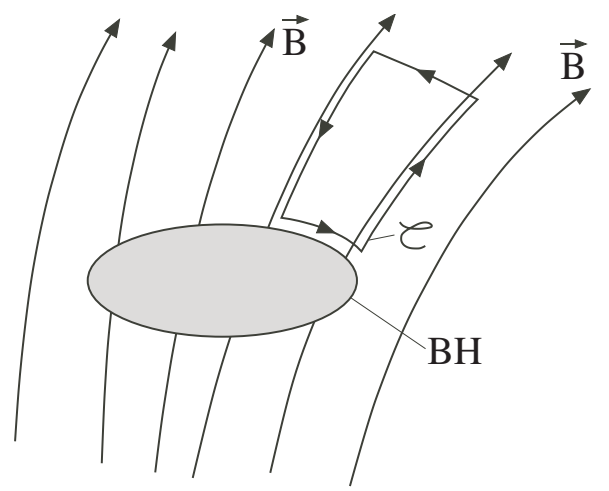

FIGURE 3. Arrangement for eq. (29).

Let us work this out for the special case of an axisymmetric field. For the closed path $\mathscr{C}$ in Fig. 3 the EMF is by (29)

$$
\mathrm{EMF} \equiv \triangle V=\int_{\mathscr{C}_{H}} i_{\beta_{H}} \mathscr{B}=-\left(-\frac{\Omega_{H}}{2 \pi}\right) \int_{\mathscr{C}_{H}} \mathbf{d} \Psi,
$$

i.e.

$$
\triangle V=\frac{\Omega_{H}}{2 \pi} \triangle \Psi
$$

This result is independent of the physics outside the black hole. 
Let us integrate it from the pole to some point north of the equator. For the exact vacuum solution in $\S 3$ we know the result for the EMF, if we integrate up to the equator: From (30) and (20) we get EMF $=2 \Omega_{H} B_{0} M\left(r_{H}-M\right)$ or $\left(\Omega_{H}=a / 2 M r_{H}\right)$

$$
\mathrm{EMF}=a B_{0} \frac{r_{H}-M}{r_{H}} \quad\left(r_{H}=M+\sqrt{M^{2}-a^{2}}\right) .
$$

Note that the "Meissner effect" for black holes implies that the EMF vanishes for extremal black holes. We shall, however, see that this property of the vacuum solution is astrophysically not relevant, because a plasma-filled magnetosphere changes the structure of the magnetic field close to the horizon dramatically. Even for a maximally rotating Kerr black hole the magnetic field is pulled inside the event horizon. For a detailed discussion we refer to [4].

For a general situation we have roughly $\Sigma \triangle \Psi=\Psi \sim B_{\perp} \pi r_{H}^{2}, \tilde{\omega}^{2} \sim<\tilde{\omega}^{2}>\sim \frac{r_{H}^{2}}{2}$. The total EMF, $V=\Sigma \triangle V$, is thus

$$
V \sim \frac{1}{2 \pi} \Omega_{H} \Psi \sim \frac{1}{2 \pi} \frac{a}{2 M r_{H}} B_{\perp} \pi r_{H}^{2} \simeq \frac{1}{2}\left(\frac{a}{M}\right) M B_{\perp}
$$

(compare this with (31)). Numerically we find

$$
V \sim\left(10^{20} \text { Volt }\right)\left(\frac{a}{M}\right) \frac{M}{10^{9} M_{\odot}} \frac{B_{\perp}}{10^{4} G} .
$$

For reasonable astrophysical parameters we obtain magnetospheric voltages $V \sim 10^{20}$ Volts. This voltage is comparable to the highest cosmic ray energies that have been detected.

Note, however, that for a realistic astrophysical situation there is plasma outside the $\mathrm{BH}$ and it is, therefore, at this stage not clear how the horizon voltage (33) is used in accelerating particles to very high energies. This crucial issue is addressed in the final section.

Let us estimate at this point the characteristic magnetic field strength than can be expected outside a supermassive $\mathrm{BH}$. A characteristic measure is the field strength $B_{E}$, for which the energy density $B_{E}^{2} / 8 \pi$ is equal to the radiation energy density $u_{E}$ corresponding to the Eddington luminosity. One finds $\left(M_{H, 8} \equiv M_{H} / 10^{8} M_{\odot}\right)$

$$
B_{E}=1.2 \times 10^{5} M_{H, 8}^{-1 / 2} \text { Gauss. }
$$

For a BH with mass $\sim 10^{9} M_{\odot}$ inside an accretion disk acting as a dynamo, a characteristic field of about 1 Tesla $\left(10^{4}\right.$ Gauss) is thus quite reasonable.

\section{BASIC EQUATIONS OF GENERAL RELATIVISTIC IDEAL MAGNETOHYDRODYNAMICS}

The relativistic fluid is described by its rest-mass density, $\rho_{0}$, the energy-mass density, $\rho$, the 4-velocity, $U^{\mu}$, and the isotropic pressure, $p$, assumed to be given by an ideal gas 
equation of state

$$
p=(\Gamma-1) \varepsilon
$$

where $\varepsilon=\rho-\rho_{0}$ is the internal energy density, and $\Gamma$ is the adiabatic index.

The basic equations of GRMHD are easy to write down. First, we have the baryon conservation

$$
\nabla_{\mu}\left(\rho_{0} U^{\mu}\right)=0 \text {. }
$$

For a magnetized plasma the equations of motion are

$$
\nabla_{v} T^{\mu v}=0
$$

where the energy-stress tensor $T^{\mu v}$ is the sum of the matter (M) and the electromagnetic (EM) parts:

$$
\begin{aligned}
T_{M}^{\mu \nu} & =(\rho+p) U^{\mu} U^{v}+p g^{\mu v}, \\
T_{E M}^{\mu \nu} & =\frac{1}{4 \pi}\left(F^{\mu}{ }_{\lambda} F^{v \lambda}-\frac{1}{4} g^{\mu v} F_{\alpha \beta} F^{\alpha \beta}\right) .
\end{aligned}
$$

In addition we have Maxwell's equations

$$
d F=0, \quad \nabla_{v} F^{\mu v}=4 \pi J^{\mu} .
$$

We adopt the ideal MHD approximation

$$
i_{U} F=0,
$$

which implies that the electric field vanishes in the rest frame of the fluid (infinite conductivity). Then the inhomogeneous Maxwell equation provides the current 4-vector $J^{\mu}$, but is otherwise not used in what follows.

As a consequence of (40) and (41) we obtain (using the Cartan identity $L_{U}=i_{U}$ ○ $\left.d+d \circ i_{U}\right): L_{U} F=0$, i.e., that $F$ is invariant under the plasma flow, implying flux conservation. The basic equations imply that

$$
* F=h \wedge U,
$$

where $h=i_{U} * F$ is the magnetic induction in the rest frame of the fluid (seen by a comoving observer). Note that $i_{U} h=0$. Furthermore, one can show that the electromagnetic part of the energy-momentum tensor may be written in the form

$$
T_{E M}^{\mu \nu}=\frac{1}{4 \pi}\left[\frac{1}{2}\|h\|^{2} g^{\mu v}+\|h\|^{2} U^{\mu} U^{v}-h^{\mu} h^{v}\right],
$$

with $\|h\|^{2}=h_{\alpha} h^{\alpha}$.

Let us also work out the $3+1$ decomposition of the ideal MHD condition (41). Using $U=\gamma\left(e_{0}+\vec{v}\right), \gamma=\left(1-v^{2}\right)^{-1 / 2}$ and the coordinate velocity $V^{i}=U^{i} / U^{t}=\alpha v^{i}-\beta^{i}$, we obtain from (5)

$$
\mathscr{E}=i_{\vec{v}} \mathscr{B} \quad \text { or } \quad \check{\mathscr{E}}=i_{\vec{V}} \mathscr{B}\left(\Rightarrow i_{\vec{V}} \check{\mathscr{E}}=0\right)
$$

Therefore, the induction equation becomes

$$
\partial_{t} \mathscr{B}+d i_{\vec{V}} \mathscr{B}=0 \quad \text { or } \quad \partial_{t} \mathscr{B}+L_{\vec{V}} \mathscr{B}=0 .
$$




\section{THE BLANDFORD-ZNAJEK PROCESS}

In this section we derive the main results obtained by Blandford and Znajek [8], by making use of the Kerr-Schild coordinates.

\subsection{Steady-state force-free magnetospheres}

BZ studied axisymmetric, force-free magnetized plasmas outside Kerr black holes. The following presentation is strongly influenced by the recent paper [9].

A plasma is said to be force-free if $i_{J} F=0$, i.e.

$$
F_{\mu \nu} J^{v}=0
$$

(no electric field in the rest system of the current). This condition follows from ideal GRMHD when the inertia of the plasma is ignored. Formally, it is obtained by letting the specific enthalpy $(\rho+p) / n$ in the ideal GRMHD equations go to zero. Since Maxwell's equations imply that $\nabla_{v} T_{E M}^{\mu \nu}=-F^{\mu}{ }_{v} J^{v}$, the energy-stress tensor of the electromagnetic field is separately conserved. The force-free condition (46) implies the constraint $F \wedge$ $F=0$, i.e., the algebraic condition $* F_{\mu \nu} F^{\mu \nu}=0$. In the literature it has often been asserted that magnetospheres of black holes should in large parts be nearly force-free This is not born out in recent simulations, except in the polar region (see Sect. 7). In 3+1 decomposition (46) becomes

$$
\rho_{e} \vec{E}+\vec{j} \times \vec{B}=0
$$

Therefore, the vector fields $\vec{E}$ and $\vec{B}$ are perpendicular.

\subsection{Energy flux at infinity}

Of particular interest is the energy flux at infinity. Since $\partial_{t}$ is a Killing field, $T_{t}^{\mu}$ are the components of a conserved 4-vector field:

$$
\frac{1}{\sqrt{-g}} \partial_{\mu}\left(\sqrt{-g} T_{t}^{\mu}\right)=0
$$

For stationary fields $\partial_{i}\left(\sqrt{-g} T_{t}^{i}\right)=0$. The electromagnetic power at infinity ${ }^{1}$ is

$$
P_{E M}=\int_{0}^{\pi} \sqrt{-g} F_{E} 2 \pi d \vartheta
$$

\footnotetext{
${ }^{1}$ In the classical BZ process one ignores the extraction of rotational energy of the black hole by accreting material interacting with the electromagnetic field. Below $T^{\mu v}$ always denotes the electromagnetic energymomentum tensor.
} 
where $F_{E}=-T_{t}^{r}$. The integral can be taken for any fixed $r$ outside the horizon, in particular at the horizon if we use Kerr-Schild coordinates. For $F_{E}$ we get

$$
F_{E}=-\frac{1}{4 \pi} F_{t l} F^{r l}=-\frac{1}{4 \pi} F_{t \vartheta} F^{r \vartheta}
$$

$\left(F_{t \varphi}=0\right)$. It is straightforward to check that $F_{E}$ is invariant under the coordinate transformation $[\mathrm{BL}] \rightarrow[\mathrm{KS}]$. Using previous results on readily finds in BL coordinates

$$
F_{E}=-\frac{1}{4 \pi} \Omega_{F} \frac{(-g)}{g_{r r} g_{\vartheta \vartheta}} \hat{B}^{r} \hat{B}^{\varphi},
$$

where $\hat{B}^{i}:=B^{i} / \alpha=* F^{i t}$.

Now we transform the result (50) to KS coordinates. It is straightforward to show that $\hat{B}^{r}, \hat{B}^{\vartheta}$ remain invariant, while the remaining component transforms as

$$
\hat{B}^{\varphi}[B L]=\hat{B}^{\varphi}[K S]-\frac{a-2 r \Omega_{F}}{\Delta} \hat{B}^{r}[K S]
$$

where $\Delta:=r^{2}-2 M r+a^{2}$. This implies that on the horizon $(\Delta=0)$

$$
\left.F_{E}\right|_{H}=\frac{1}{2 \pi}\left(\hat{B}^{r}\right)^{2} \Omega_{F} r_{H}\left(\Omega_{H}-\Omega_{F}\right) \sin ^{2} \vartheta .
$$

Therefore,

$$
P_{E M}=\int_{0}^{\pi} d \vartheta \rho_{H}^{2} \sin \vartheta\left(\hat{B}^{r}\right)^{2} \Omega_{F} r_{H}\left(\Omega_{H}-\Omega_{F}\right) \sin ^{2} \vartheta
$$

or in terms of the normalize normal component $B_{\perp}$

$$
P_{E M}=\frac{1}{2} \int_{0}^{\pi} d \vartheta \Omega_{F}\left(\Omega_{H}-\Omega_{F}\right) \rho_{H} \tilde{\omega}^{3} B_{\perp}^{2}
$$

The important result (54) shows that $P_{E M}$ becomes maximal for $\Omega_{F} \approx \frac{1}{2} \Omega_{H}$. Then

$$
P_{E M}^{\max }=\frac{1}{8} \Omega_{H}^{2} \int_{0}^{\pi} \rho_{H} \tilde{\omega}^{3} B_{\perp}^{2} d \vartheta
$$

After angular integration one finds, if $B_{\perp}^{2}$ is replaced by an average value $\left\langle B_{\perp}\right\rangle^{2}$,

$$
P_{E M}^{\max }=\frac{1}{4}\left(\frac{a}{M}\right)^{2} M^{2}\left\langle B_{\perp}\right\rangle^{2} f\left(\frac{a}{r_{H}}\right),
$$

where the function $f$ is not far from 1 [10] . Numerically,

$$
P_{E M}^{\max }=1.7 \times 10^{46} \frac{\mathrm{erg}}{\mathrm{s}}\left(\frac{a}{M} \cdot \frac{M}{10^{9} M_{\odot}} \cdot \frac{\left\langle B_{\perp}\right\rangle}{10^{4} G}\right)^{2} f .
$$

For the angular momentum flux $F_{L}$ one finds $F_{E}=\Omega_{F} F_{L}$. Using this one can show that up to $9 \%$ of the initial mass can, in principle, be extracted [10].

Whether the crucial condition $\Omega_{F} \approx \frac{1}{2} \Omega_{H}$ is approximately satisfied in realistic astrophysical scenarios is a difficult problem for model builders. This brings me to recent numerical work by several groups. 


\section{GENERAL RELATIVISTIC MHD SIMULATIONS}

In recent years several groups have developed codes for ideal GRMHD, and applied them in particular for studies of the generic BZ mechanism. These numerical studies show how accretion dynamics self-consistently create large scale magnetic fields and explore the resulting outflows.

\subsection{Numerical methods}

There are many ways to write the basic equations in a form suitable for numerical integration. A pioneering code, based on the $3+1$ splitting relative to the FIDO tetrad described in Sect. 2, has been developed by Koide and collaborators (for a detailed description, see [11]). The system to be integrated consists of eight evolutionary equations for two scalar and two vector quantities (primary code variables), which contain in addition five 'primitive' variables that can be determined from the former by solving two coupled polynomial equations $P(x, y)=0, Q(x, y)=0$. This process, like similar iterative root-finding processes in other schemes, is time consuming.

A code that is able to perform long-term (several thousand of $M$ in time) GRMHD simulations, has been developed by Villiers \& Hawley [12]. It evolves different auxiliary variables from which the primitive variables are easily recovered. In contrast to some alternative schemes [13], [14], it is not fully conservative. Since BL coordinates are used, the inner boundary condition must lie outside the horizon. KS coordinates are used in the axisymmetric code HARM [13]. In a modified version of this [19] the inversion from "conserved" quantities to "primitive" variables has been improved (see Appendix A).

A specific common problem is that the magnetic field is advanced in time by an antisymmetric differential operator, and not by a differential operator of divergence form. In addition one has to guarantee that the numerical scheme preserves the constraint equation $d \mathscr{B}=0$ to rounding error. One way to handle this problem is the so-called constraint transport (CT) method, where the induction equation is discretized such that the solenoidal constraint is built in [15].

No code is perfect, and it is therefore important that the outcomes of different approaches are compared.

\subsection{Qualitative results}

I first summarize some of the results presented in [16] on simulations of accretion flows on rotating black holes, and the properties of the resulting unbound outflows ${ }^{2}$.

The qualitative late-time structure is illustrated in Fig. 4. Along the equator there is a wedge-shaped Keplerian accretion disk and a net accretion flow is produced by MHD turbulence whose origin is a magneto-rotational instability, which leads to a

2 This is based on a sequence of earlier papers on the the subject, cited in [16] 
fast amplification of the magnetic field (see Appendix B). A short distance outside the marginally stable circular stable orbit, the equatorial pressure and density reach a local maximum in a 'inner torus' region. Inside this local pressure maximum, the density and pressure drop as the flow accelerates toward the black hole in the 'plunging region'. Above and below the disk is a 'corona' of hot magnetized plasma with a magnetic field whose typical strength is near equipartition. Along the spin axis of the $\mathrm{BH}$ there is a 'centrifugal funnel' that is largely empty of matter, but filled with magnetic field and an outward Poynting flux. Between the evacuated funnel and the corona there is a region of unbounded mass flux, referred to as the 'funnel wall jet'. It is this part that is the center of attention in [16]. Of particular interest is the strength of the jet compared to the amount of accretion on the BH. The quantification of this is somewhat ambiguous, because matter and electromagnetic fluxes are usually not constant in time or radius, and there is exchange between the two components.

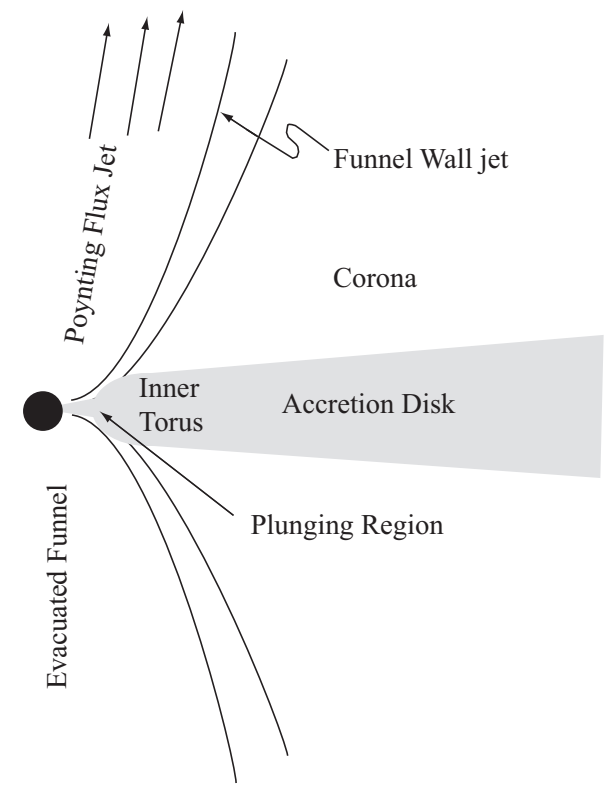

FIGURE 4. Main dynamical features in accretion disk simulations. Fig. 1 of [16].

The numerical results show that for rapidly-rotating BH's the jet plays a significant role in the energy budget. When $|a / M| \geq 0.9$, the total jet efficiency is generally several tens of percent, with the matter portion somewhat larger than the electromagnetic part. The ratio of angular momentum in the unbound outflow to that deposited in the $\mathrm{BH}$ is also a very strong function of $\mathrm{BH}$ spin. In the non-rotating case this is only a few tenths of a percent, but rises to $\simeq 25 \%$ when $a / M=0.9$. For $a / M=0.99$, the highest spin case reported in [16], the rate at which the $\mathrm{BH}$ spins down due to electromagnetic torques nearly matches the rate at which it acquires angular momentum by accreting matter. Indeed, the angular momentum expelled in the outflow is an order of magnitude larger than the net amount captured. In all cases, the electromagnetic portion of the angular momentum carried away is comparable to the matter portion carried in the funnel wall jet.

It has to be emphasized that in the initial state of the simulations there is no magnetic field in the region that eventually becomes the outflow region. The large scale 
magnetic field within the funnel and the Poynting flux jet rapidly develop as a result of magnetically controlled accretion. The formation can be described as follows. When disk material reaches the horizon, strong magnetic pressure gradients are built up which drive the plasma upward. In turn, this motion drains off the field lines, forming a "magnetic tower". This was previously seen in non-relativistic simulations using a pseudoNewtonian potential [18]. It is interesting that the evacuated funnel is the only region

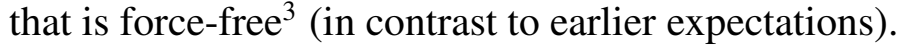

The matter-dominated outflow moves at a modest velocity $(v / c \sim 0.3)$ along the centrifugal barrier surrounding the evacuated funnel. The funnel wall jet turns out to be accelerated and collimated by magnetic and gas pressure forces in the inner torus and the surrounding corona. The magnetic field is spun by the rotating Kerr spacetime, hence the energy of the Poynting flux jet comes from the $\mathrm{BH}$ rotation. Below we will address the question whether magnetic forces might provide additional acceleration and collimation on far larger scales than are modeled in [16].

Hawley and Krolik conclude from their numerical studies that while the proximate energy source is the BH's rotation, accretion replenishes both the BH's mass and angular momentum. A substantial decrease of the rotational energy of the BH does not appear to be a generic phenomenon. This is in contrast to the classic BZ model. For a detailed discussion, I refer to the original paper.

Similar results were found before by McKinney and Gammie in their axisymmetric simulations [9]. Among other aspects these also showed that as the hole loses energy and angular momentum, its total mass and angular momentum are replenished by accretion. In [20] McKinney followed the evolution of the jet till $t \approx 10^{4} G M / c^{3}$ out to $r \approx$ $10^{4} G M / c^{2}$, and found that by then the jet has become superfast magnetosonic and moves at a Lorentz factor of about 10 . This may, however, only be a small fraction of the Lorentz factor at much larger distances. Indeed, if a large fraction of the magnetic and thermal energy would go into particle kinetic energy, one would estimate from the simulation that the terminal Lorentz factor may reach almost $10^{3}$. This estimate is based on the following fact. For a stationary axisymmetric flow the energy (momentum) flux per unit rest-mass flux is conserved along flux surfaces and can thus be determined from local flow quantities. The relevant formula for this quantity is, using previous notation,

$$
\frac{-T_{t}^{A}}{\rho_{0} U^{A}}=-\frac{\rho+p}{\rho_{0}} U_{t}-\frac{\Omega_{F}}{4 \pi} \frac{\hat{B}^{A}}{\rho_{0} U^{A}} \check{\mathscr{H}}_{\varphi} \quad(A=r, \vartheta) .
$$

Since the simulation in [20] stopped long before the end of the acceleration period, Komissarov et al. have studied numerically the further evolution of the relativistic jet [21]. Key issues of this investigation are: (i) Is the magnetic driving mechanism able to accelerate outflows to high Lorentz factors with high efficiency over astrophysically extended scales? (ii) Can these flows be collimated by purely magnetic stresses? Since most of the acceleration takes place far away from the black hole, the simulations are carried out in the framework of special-relativistic ideal MHD.

\footnotetext{
${ }^{3}$ The criterion for the validity of this property is that $\left(\|h\|^{2} / 8 \pi\right) /(\rho+p) \gg 1$.
} 
The authors investigate models of the following kind. The free boundary with an ambient medium is replaced by solid rigid walls on which appropriate boundary conditions are imposed. This simplification enables higher numerical accuracy. At the inlet boundary, the injected poloidal current distribution is prescribed through a rotational profile. The initial configuration corresponds to a non-rotating purely poloidal magnetic field with nearly constant magnetic pressure across the funnel. Moreover, the outflows are initially Poynting flux-dominated. The authors find that these approach a steady state with a spatially extended acceleration region. Furthermore, the acceleration process turns out to be very efficient; almost $80 \%$ of the Poynting flux is converted into kinetic energy. The results also show efficient self-collimation. In contrast to [20], no instabilities or shocks are found in the simulation.

We have already remarked earlier that even for very rapidly rotating black holes the Blandford-Znajek process can drive magnetized jets. Fig. 5 from [4] shows clearly that there is no "Meissner effect" at work. This is due to the fact that within the ergosphere the plasma unavoidably corotates with the black hole.

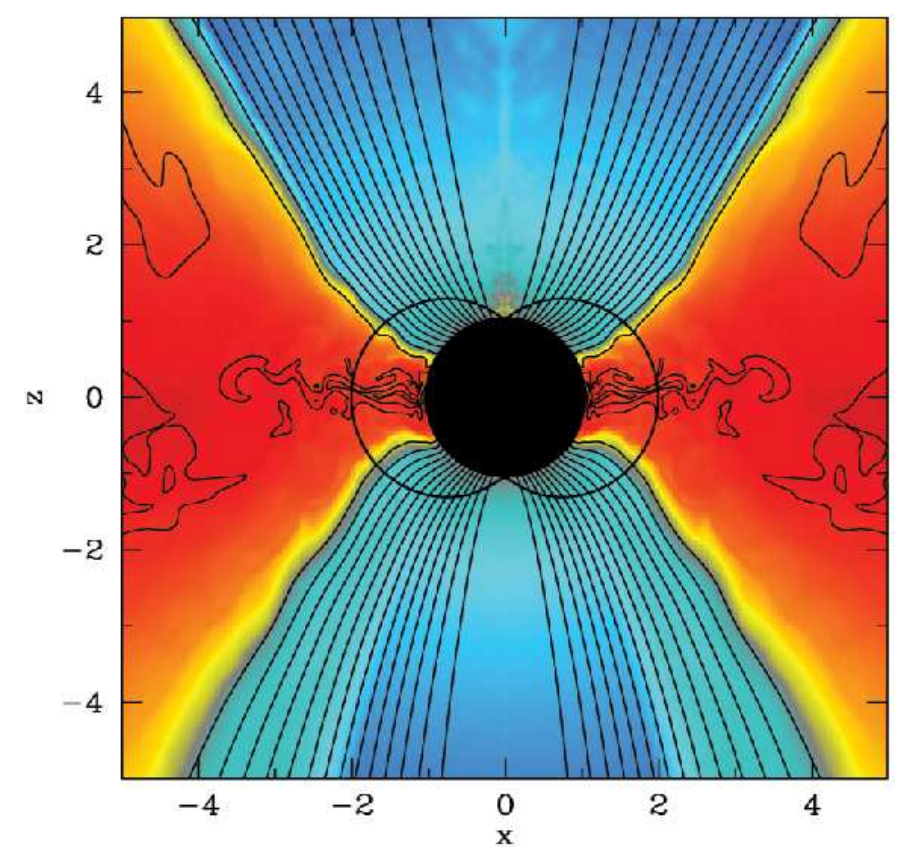

FIGURE 5. Magnetic field lines and logarithm of rest-mass density (colored) quasi-steady accretion disk simulations for an almost extreme Kerr black hole $(a / M=0.999)$ [Fig. 3 of [4]].

One of the main shortcomings of existing simulations is that the radiation field is completely neglected. It is likely that radiative and other high energy processes play a significant role in the flow dynamics through radiation force on the outflowing plasma. Moreover, at some point physical resistivity will also have to be included, and the singlefluid approximation will not always hold everywhere. Numerical calculations will be performed in 3D. 


\section{A. NUMERICAL SCHEMES FOR GRMHD}

Most GRMHD codes have adopted a conservative scheme, which means that the integrated evolutionary equations are of the general form

$$
\partial_{t} \underline{U}(\underline{P})+\partial_{i} \underline{F}^{i}(\underline{P})=\underline{S}(\underline{P}) .
$$

Here, $\underline{U}$ are vector-valued "conserved" variables, $\underline{P}$ is a vector of "primitive" variables (rest-mass density, internal energy density, velocity components and magnetic field components). The fluxes $\underline{F}^{i}, \underline{U}$ and the "source vector" $\underline{S}$ depend on $\underline{P}$. Conservative numerical schemes advance $\underline{U}$, then calculate $\underline{P}(\underline{U})$ once or twice per time step.

While in praxis the map $\underline{P} \mapsto \underline{U}(\underline{P})$ is analytically known, the inverse map $\underline{U} \mapsto \underline{P}(\underline{U})$ is not available in closed form and must be computed numerically. How this is performed is at the heart of a conservative scheme, since the operation must be accurate, fast and robust.

Below we describe these points in more detail, following [19]. We use the notation and basic equations introduced in Sect. 5 .

Equations (36), (37) and (45) can readily be written in the form (59) in terms of the eight conserved variables

$$
\underline{U}: \quad D:=\gamma \rho_{0}, Q_{\mu}:=-u_{v} T_{\mu}^{\nu}, \vec{B},
$$

where $\gamma=-u_{\mu} U^{\mu}=\left(1-v^{2}\right)^{-1 / 2}$. As eight primitive variables we use

$$
\underline{P}: \rho_{0}, \varepsilon, \vec{B}, v^{i}
$$

(The vector $\vec{B}$ is common to $\underline{U}$ and $\underline{P}$.)

In [19] it is shown that $W:=\rho_{0} h \gamma^{2}$, where $h:=1+\varepsilon+p / \rho_{0}$, and $v^{2}$ can be determined from $\underline{U}$ by solving two polynomial equations. After that one can easily recover all primitive variables.

\section{B. MAGNETO-ROTATIONAL INSTABILITY}

This important instability, whose crucial astrophysical implications have been understood astonishingly late, shows up already in linearized theory. For a detailed pedagogical discussion we refer to [17].

In a systematic treatment one linearizes the basic equations of MHD for small fluctuations of a disk system, consisting of a central point mass and a differentially rotating magnetized disk. An analysis of the resulting rather complicated dispersion relation leads to the following results. In the non-rotating limit (homogeneous unperturbed situation) one finds the familiar Alfvén waves and two other MHD modes. The fast one is often referred to as magnetosonic wave, and represents magnetic and thermal pressure in concert. In the slow mode magnetic tension and gas compression act in opposition. For weak fields it becomes degenerate with the Alfvén mode, while for strong fields it becomes an ordinary sound wave, channeled along the field lines. 
The effect of Kepler rotation on these three modes is very interesting: At a critical rotation frequency the slow MHD mode becomes unstable.

There is a simple way to understand this far reaching instability. Consider an axisymmetric gas disk in the presence of a vertical magnetic field, that has no effect on the disk equilibrium. Assume that a fluid element is displaced from its circular orbit by $\xi \propto e^{i k z}$ ( $z=$ vertical direction). Using the induction law and simple mechanics one finds the these incompressible planar displacements satisfy the same equation as the separation of two orbiting mass points, connected by a spring (with a spring constant related to the Alfvén velocity). It is quite obvious that this system is unstable; the separation of the two mass points rapidly increases. This is the essence of the weak-field magneto-rotational instability.

The magneto-rotational instability plays a fundamental role in disk accretion, because it leads to disk-turbulence and corresponding stresses.

\section{ACKNOWLEDGMENTS}

I wish to thank the organizers of the Symposium on Gravitation and Cosmology, in particular C. Laemmerzahl and A. Macias, for inviting me and their wonderful hospitality. I am grateful that J.C. McKinney and S.S. Komissarov allowed me to include two figures of their work in this article.

\section{REFERENCES}

1. K. S. Thorne, R. H. Price \& D. A. MacDonald, Black Holes: The Membrane Paradigm, Yale Univ. Press. (1986).

2. R. Durrer \& N. Straumann, Helv. Phys. Acta. 61, 1027 (1988).

3. R. M. Wald, Phys. Rev. D 10, 1680 (1974).

4. S. S. Komissarov and J. C. McKinney, astro-ph/0702269.

5. K. S. Thorne \& D. A. MacDonald, Mon. Not. Roy. Astron. Soc. 198, 339 (1982).

6. N. Straumann, General Relativity, With Applications to Astrophysics, Texts and Monographs in Physics, Springer Verlag, 2004.

7. N. Straumann, The Membrane Model of Black Holes and Applications. In: F.W. Hehl, C. Kiefer and R.J.K. Metzler (eds.), Black Holes: Theory and Observation. Springer-Verlag 1998; astro-ph/9711276.

8. R. D. Blandford \& R. L. Znajek, Mon. Not. Roy. Astron. Soc. 179, 433 (1977).

9. J. C. McKinney and Ch. F. Gammie, Astrophys. J. 611, 977 (2004).

10. H. K. Lee, R. A. Wijers and G. Brown, Physics Reports 325, 83 (2000).

11. S. Koide, Phys. Rev. D 67, 104010 (2003).

12. J.-P. De Villiers \& J. F. Hawley, Astrophys. J. 589, 458 (2003).

13. Ch. F. Gammie, J. C. McKinney \& G. Tóth, Astrophys. J. 589, 444 (2003).

14. S. S. Komissarov, MNRAS 350, 1431 (2004).

15. C. R. Evans \& J. F. Hawley, Astrophys. J. 332, 659 (1988).

16. J. F. Hawley \& J. H. Krolik, Astrophys. J. 641, 103 (2006) [astro-ph/0512227].

17. S. A. Balbus \& J. F. Hawley, Rev. Mod. Phys. 70, 1 (1998).

18. Y. Kato, S. Mineshige \& K. Shibata, Astrophys. J. 605, 307 (2004).

19. S. C. Noble, C. F. Gammi, J. C. McKinney, L. D. Del Zanna Astrophys. J 641, 626 (2006) [astro$\mathrm{ph} / 0512420]$.

20. J. C. McKinney, Mon.Not.R.Astron.Soc. 368, 1561 (2006) [astro-ph/0506369].

21. S. S. Komissarov, M. V. Barkov, N. Vlahakis and A. Königl, astro-ph/0703146. 